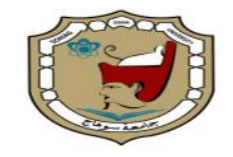

Sohag University

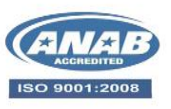

Sohag Medical Journal

Faculty of Medicine

\title{
Role of Magnetic Resonance Imaging and Magnetic Resonance Spectroscopy in the diagnosis of Neonatal Hypoxic-Ischemic Injury.
}

\author{
Barakat M. Mahmoud, Mohammad T. Mahmoud, \\ Mohammad Z. Ali, Khaled F Zaky
}

Department of Diagnostic Radiology, Faculty of Medicine, Sohag University

\begin{abstract}
Background: Hypoxic Ischemic Encephalopathy is defined as a heterogeneous, clinically defined syndrome characterized by disturbed neurologic function in the earliest days of life in a neonate. Proton MR spectroscopy could reveal brain ischemic injuries in asphyxiated neonates earlier than T1- or T2-weighted MR imaging. MR spectroscopy and MR imaging are very important for the diagnosis of hypoxic-ischemic injury.

Patients and Methods: A cross-sectional study. From December 2018 to December 2019, 50 full-term neonates were admitted to Neonatal Care Unit (NCU) in Sohag University Hospital with signs of HIE and referred to the Radio Diagnosis Department for brain imaging. MR imaging studies were performed using an MRI system 1.5 tesla Achieva, Philips superconductive MRI system. Our MRI sequences 1. Axial T1WI. 2. Axial T2WI. 3. Axial FLAIR. 4. Axial DWI. 5. proton MR spectroscopy

Results: The study included 50 neonates; 7 of them had normal MRI findings. The other 43 neonates showed different patterns of injury; 24 neonates showed a central pattern of injury, 11 neonates show peripheral patterns of injury, While the other 8 neonates had shown both central and peripheral patterns of brain injury. Only 45 of the examined neonates show abnormal lactate peak. The sensitivity of MRS is $100 \%$. However, the specificity is low $70 \%$, the overall accuracy is $60 \%$.

Conclusion: MR spectroscopy is an accurate, sensitive, and non-invasive method for early detection of perinatal ischemic brain injuries.

Keywords: MRS, MRI, NHIE.

\section{Introduction}

Hypoxic Ischemic Encephalopathy (HIE) is defined as a heterogeneous, clinically defined syndrome characterized by disturbed neurological functions in the earliest days of life in a newborn at or beyond 35 weeks of gestation, presented by a reduced level of consciousness convulsions, and often accompanied by respiratory problems, and by depression of tone and reflexes (1). Acute HIE due to perinatal hypoxia-ischemia is one of the leading causes of neonatal mortality worldwide. Hypoxic-ischemic encephalopathy is a brain injury secondary to inadequate blood flow to the infant's brain occurring because of a hypoxic-

intrapartum, or postnatal period. Neonatal encephalopathy results from a wide variety of conditions as birth asphyxia (1). Head computed to mogramp, ultrasound, and conventional magnetic resonance imaging (MRI) are helpful to diagnose brain injury but cannot quantify the degree of white matter damage $(\underline{2}, \underline{3})$. Conventional MRI reveals that the two types of brain injury in HIE neonates are basal ganglia, thalamic abnormalities, and watershed dam-age, which are strongly associated with late motor and cognitive deficits $(\underline{2}, \underline{3})$.
\end{abstract} ischemic insult during the prenatal, 
MRI serves as an important role in imaging the neonatal brain. MRS allows real-time analyses of the metabolites in the neonatal brain. The potential utility of proton MR spectroscopy could reveal brain ischemic injuries in asphyxiated neonates earlier than T1- or T2weighted MR imaging (4-7).

\section{Aim of the work:}

Assess the role of MR spectroscopy and MR imaging in the diagnosis of hypoxicischemic injury.

\section{Patients and Methods:}

Study design: A cross-sectional study Patients: From December 2018 to December 2019, 50 full-term neonates $\{32$ males $(64 \%)$ and 18 females $(36 \%)\}$ were admitted to the Neonatal Care Unit (NCU) in Sohag University Hospital with signs of HIE and referred to the Radio Diagnosis Department for brain imaging.

\section{Inclusion criteria:}

1- Full-term neonates (37-42 weeks' gestational age).

2- Patients exposed to perinatal hypoxic conditions based on the history of difficult or prolonged labor, obstructed labor, placenta previa, cord prolapse, and/or severe hemorrhage.

\section{Exclusion criteria:}

1- Pre-term neonates (less than 37 weeks gestational age).

2- Neonates with metabolic diseases and neonatal sepsis.

3- Kernicterus.

4- Traumatic birth injuries.

5- Congenital brain malformations.

We use two grading systems for clinical assessment of HIE as

1) Sarnat score; It correlates the EEG findings to the clinical condition of the infant into stage I (mild), stage II (moderate), stage III (severe) HIE.

2) The Thompson score; Depend on the grading of encephalopathy signs. This scoring system has been used widely than the Sarnat score as it depends on neurological outcomes during the first hours of life.

\section{Methods:}

All patients were suspected to full history taking and clinical examination immediately before imaging and clinical examination after imaging at the time of discharge. Approval for this study was obtained from the Research Ethics Committee of Sohag Faculty of Medicine. All study procedures were carried out under the Declaration of Helsinki regarding research involving human subjects considering patient and methods.

MR imaging studies were performed using an MRI system 1.5 tesla Achieva, Philips superconductive MRI system in Sohag university hospital. Brain coil was used in cases done with this device.

\section{The technique of MR imaging:}

All patients come cooled from Sohag Hospital Pediatric Neonatal Unite. All patients were laid supine with the head in a neutral position provide that the patient is sleeping. No movement was allowed during the examination as the patient had taken hypnotic as chloral hydrate from the oral route half an hour before the study started.

\section{MRI sequences}

- Axial T1WI.

- Axial T2WI.

- Axial FLAIR.

- Axial DWI.

- Coronal T2W1

- Proton MR spectroscopy.

\section{MRI Acquisition protocol}

\section{a. Conventional MRI protocols;} included T1-weighted sequences (TR 6.6, TE 2.6, TI 600, NEX 1, flip angle 25 , matrix $192 \times 192,1.2 \mathrm{~mm}$ slice thickness, 0 mm gap). T2- weighted axial sequences (TR 4400, TE 120, NEX 2, Matrix 384 x 320, 3 mm slice thickness, $1.0 \mathrm{~mm}$ gap), and Diffusion-weighted imaging (DWI) with single shot, b value $=1,000 \mathrm{~mm} 2 / \mathrm{s},(\mathrm{TR} 8000, \mathrm{TE} 78.8$, NEX 1, field of view (FOV) $220 \times 220$, matrix $140 \times 140$, slice thickness 2.5 $\mathrm{mm}$ with $0.6 \mathrm{~mm}$ gap). 
b. $\boldsymbol{H}$-MRS performed by multivoxel intermediate-echo sequence (TE, $144 \mathrm{~ms}$, TR, $1000 \mathrm{~ms} ; 12$ x 12 phase-encoding steps; 128 signals acquired; 2 NEX, 15$\mathrm{mm}$ slab thickness; and a nominal voxel size of $1.125 \mathrm{~cm}^{3}$ ). MRS acquisition took 8 minutes. We measure the following metabolites 1-Lactate as well as its ratio to Creatine (Lac/Cr ratio) 2 NAA and its ratio to choline (NAA/Ch ratio 3-Ratio of NAA to Last.

\section{Statistical Analysis;}

\begin{tabular}{|c|c|c|c|}
\hline \multicolumn{2}{|c|}{} & No & Percent \\
\hline Sarnat Staging & I & 23 & 46.0 \\
\cline { 2 - 4 } & II & 20 & 40.0 \\
\cline { 2 - 4 } & III & 7 & 14.0 \\
\cline { 2 - 4 } & Total & 50 & 100.0 \\
\hline
\end{tabular}

\section{Table (1): Sarnat staging}

c. Data were collected and entered to the computer using SPSS (Statistical Package for Social Science) program for statistical analysis (ver 21). Data were entered as numerical or categorical, as appropriate. KolmogorovSmirnov test of normality revealed significance in the distribution of some variables, so the non-parametric statistics was adopted.

d. Data were described using minimum, maximum, mean, standard deviation, and $95 \% \mathrm{CI}$ of the mean for the normally distributed data. Data were described using minimum, maximum, median, and interquartile range for not-normally distributed data. Comparisons were carried out between two studied independent not-normally distributed subgroups using the Mann-Whitney U test.

\section{Results}

The study included 50 neonates; 32 (64 $\%)$ were males while the remaining 18 (36\%) were females. The gestational age at birth was 37-41 weeks (mean, 37.9 weeks) and the average birth weight was 3000-4000 gm (mean, 3.7 $\mathrm{kg}$ ). At the time of the MRI examination, the mean age of the neonates was 19.82 days. The mean age of the study group was 19.8 days, with a range from 6 days to one month (30 days). Two-thirds of the cases $(64 \%)$ were males, and $36 \%$ were females. The mean gestational age of the study group was 37.92 weeks, with around $40 \%$ of the cases had 38 weeks' gestational age. The mean weight of the study neonates was $3.7 \mathrm{Kg}$, with a range of $3-4 \mathrm{Kg}$. The vast majority of our cases $(78 \%)$ were from rural areas, while only $22 \%$ were from urban regions. The majority of cases $(72 \%)$ had a positive history of obstetric problems. Among the studied neonates, 23 cases (46\%) were

classified as Sarnat stage I or mild degree HIE according to Thompson score, 20 cases were Sarnat stage II or moderate degree, while the remaining 7 cases were Sarnat stage III or severe degree HIE as shown in Table 1.

\section{Table (1): Sarnat staging}

Around half of the cases $(46 \%)$ had a mild Thompson score, $40 \%$ had a moderate score and $14 \%$ had a severe score. 50 neonates; 7 of them had normal MRI findings with normal neurological outcome. The other 43 neonates showed different patterns of injury; 24 neonates showed a central pattern of injury ( $\mathrm{T} 1$ hyperintensity of the putamina and thalami (figure1) with loss of T1 hyperintensity of the posterior limbs of the internal capsules (figure 2) and some cases had shown decreased signal intensity of both thalami in T2WI), 11 cases of them had normal outcome at the time of early discharge and 13 neonates had an abnormal neurological outcome. We found that 11 neonates showed a peripheral pattern of injury (cortical, subcortical white matter and or periventricular white matter hyperintense signal in T2W1, FLAIR and or DWI as in figure (3); 5 cases show restriction of diffusion in DWI and presented in early time 
(Within first 10 days) with or without smudging of the grey-white matter interface at the watershed regions), 3 neological outcome at the time of discharge. While the other 8 neonates had shown both central and peripheral patterns of brain injury and all of them had an abnormal outcome. $64 \%$ of the cases had central brain injury; either alone

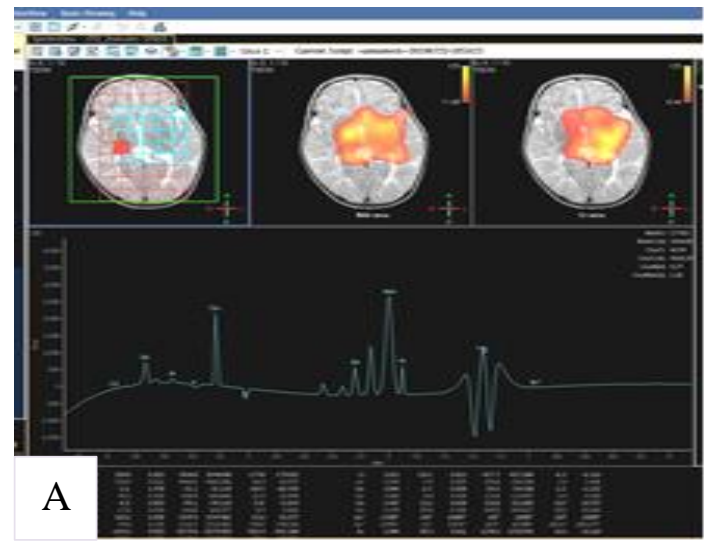

nates of 11 had normal neurological outcome at the time of discharge and other 8 neonates had abnormal neuro$(48 \%)$ or combined with peripheral injury (16\%); while peripheral injury alone was seen in $22 \%$ of the cases. Seven cases $(14 \%)$ had no brain injury with normal MRI findings.
B

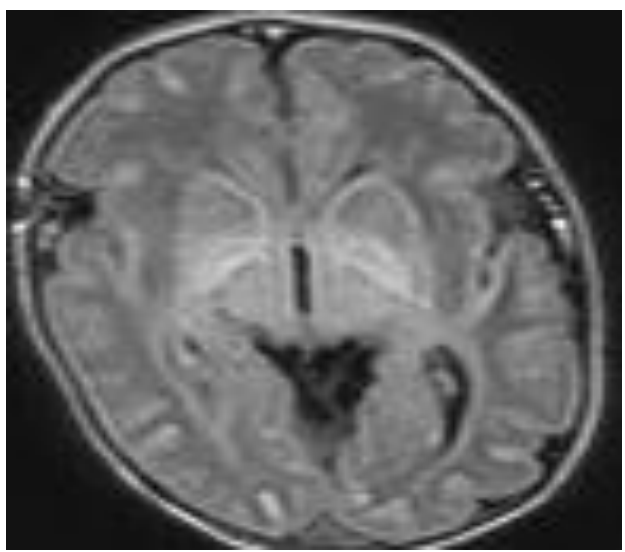

Figure (1); A) MRS shows an intermediate lactate peak. B) Axial T1W1 in 7 days' neonate with severe signs of HIE, noted bilateral thalamic and basal ganglia hyperintensity signal (Central type of brain injury).
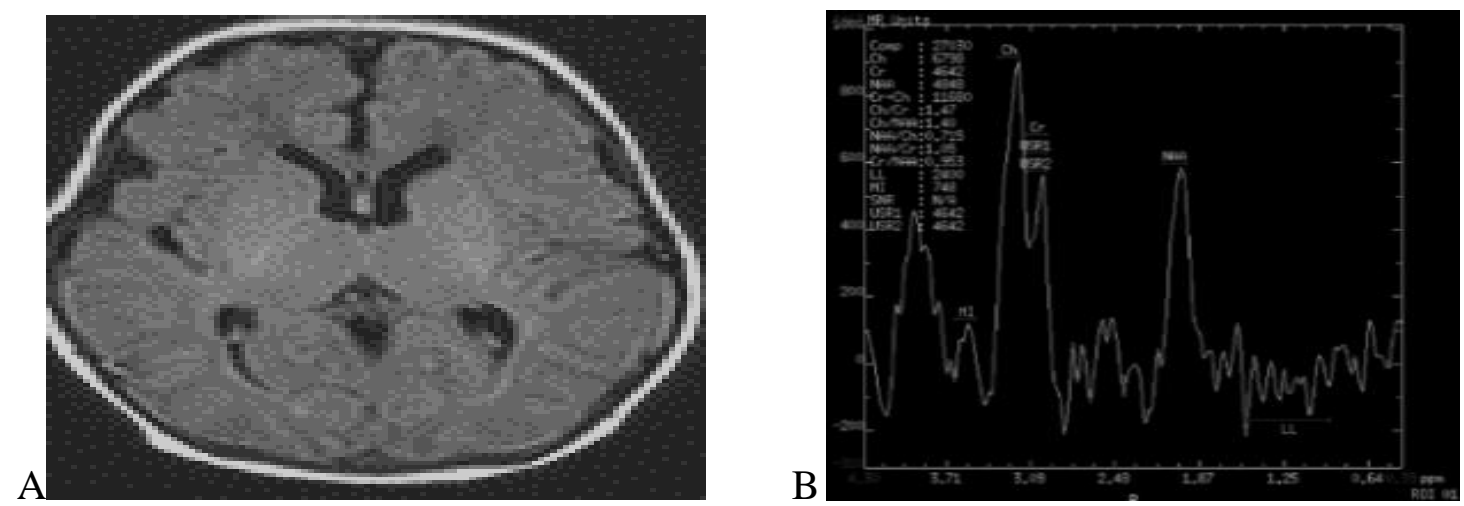

Figure (2); A) Axial T1W1 in 25 days' neonate with mild signs of HIE, noted the mild bilateral decreased signal intensity of posterior limbs of internal capsules B) MRS shows low lactate peak (Central type of brain injury).

We found 5 cases with normal MRS; No lactate peaks could be seen; Normal high level of NAA, normal choline, and creatine levels; we found 19 cases with mild HIE (low lactate peak); Normal NAA, normal choline and creatine levels. We found 19 cases with moderate HIE (intermediate lactate peak), Mild decreased NAA. We found
7 cases with severe HIE (high lactate peak), decreased NAA significantly, and decreased NAA/choline ratio. In injured newborns with HIE, a large quantity of lactate is typically present demonstrates a case of an HIE patient born at term with elevated lactate levels across the brain. 

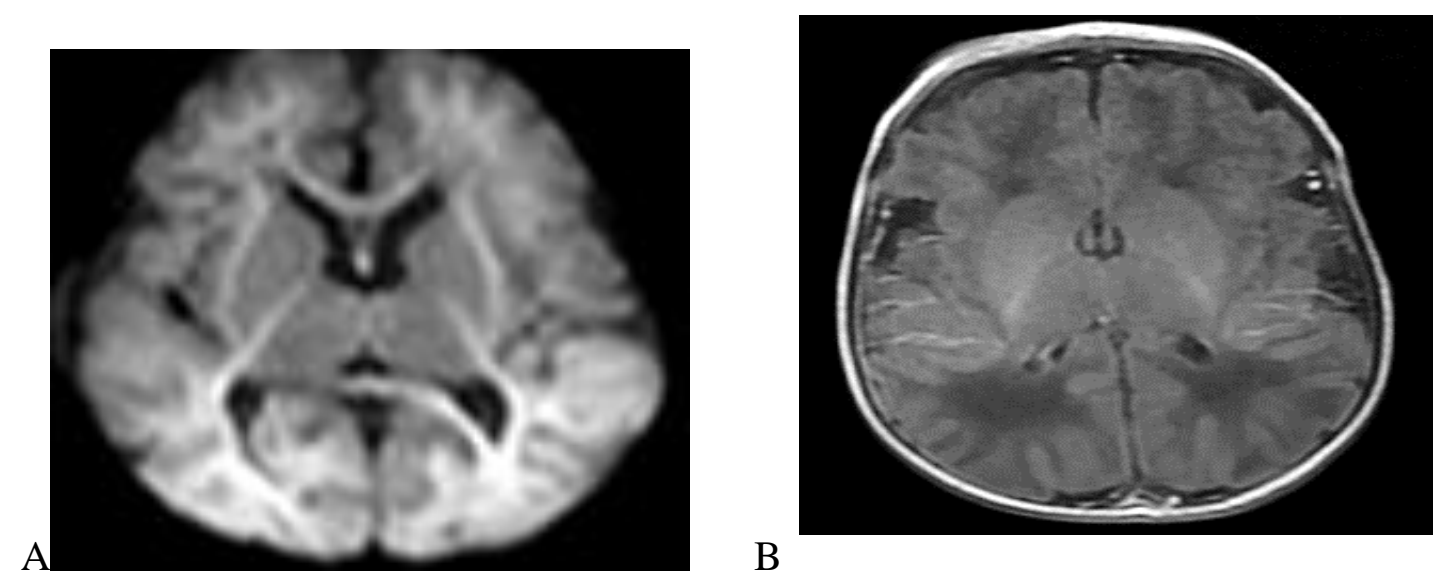

Figure (3); A) Diffusion-weighted MR in 12 days' neonate presented with moderate HIE, Sarnat score II with abnormal neurological outcome, noted bilateral occipito-parietal cortical and subcortical white matter abnormal hyperintense signal B) Axial T1W1 shows preserved PLIC (peripheral pattern of brain injury).

Only 45 of examined neonates show abnormal lactate peak; Low peak, moderate peak or high peak, reduced NAA, and elevated choline (figure 4), Although normal neonatal MRS usually reveals mildly elevated choline and reduced NAA due to incomplete myelination process. We found that $48 \%$ of the cases had abnormal neurological status at the time of discharge. $100 \%$ of cases with abnormal outcome showed abnormal MRI spectroscopic findings, compared to $80.8 \%$ among those with the normal outcome. The difference was statistically significant with a p-value of 0.031 . Nearly all cases $(90 \%)$ had abnormal findings on MRI spectroscopy as shown in table $2.48 \%$ of the cases had abnormal neurological status at the time of discharge. $100 \%$ of cases with abnormal outcomes showed abnormal MRI spectroscopic findings, compared to $80.8 \%$ among those with the normal outcome. The difference was statistically significant with a p-value of 0.031 . There was a highly significant relationship between the type of brain injury and outcome, with a p-value of less than 0.001. Among those with the abnormal outcome, $54.2 \%$ had a central brain injury, $33.3 \%$ had both central and peripheral injuries, 12.5 had only peripheral injuries; while non of them had a normal brain. On the other hand, among those with the normal outcome, $42.3 \%$ had a central brain injury, $30.8 \%$ had a peripheral injury, $26.9 \%$ had a normal brain, and non-had combined central and peripheral injuries. The sensitivity of MRI to predict a poor outcome is $100 \%$. However, the specificity is very low (19.23\%), the positive and negative predictive values are $53.33 \%$ and $100 \%$; respectively. Finally, the overall accuracy is $59.62 \%$. 


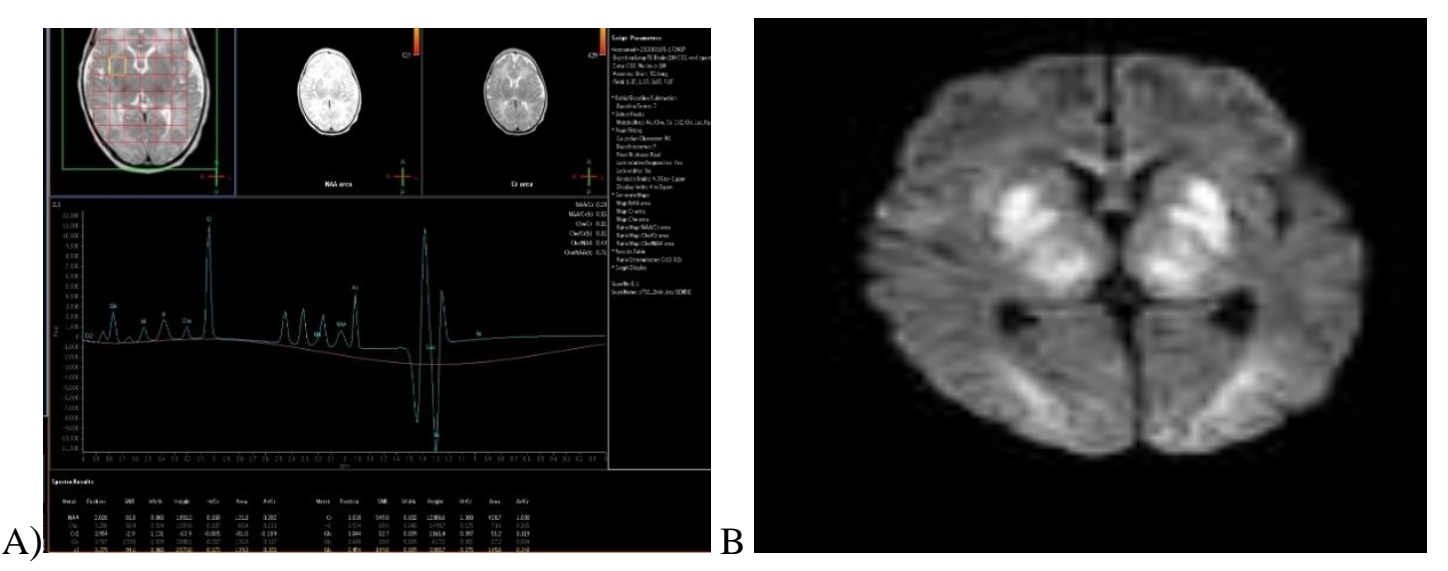

Figure (4); A) MRS in 10 days' neonate presented with severe HIE, Sarnat scores III with abnormal neurological outcome, noted marked decreased NAA with large inverted peak Lactate. B) DWI shows bilateral basal ganglia marked hyperintense signal.

\begin{tabular}{|l|l|l|l|}
\hline \multicolumn{2}{|c|}{} & No & Percent \\
\hline $\begin{array}{l}\text { MRI } \\
\text { spectroscopy } \\
\text { findings }\end{array}$ & Normal & 5 & 10.0 \\
\cline { 2 - 4 } & Abnormal & 45 & $\mathbf{9 0 . 0}$ \\
\cline { 2 - 4 } & Total & $\mathbf{5 0}$ & 100.0 \\
\hline
\end{tabular}

Table (2): MRI spectroscopy findings;

Table 3 shows that the sensitivity of central brain injury to predict a poor outcome is $87.5 \%$; the specificity is $57.69 \%$, the positive and negative predictive values are $65.63 \%$ and $83.33 \%$; respectively. Finally, the overall accuracy is $72.6 \%$.

\begin{tabular}{|l|l|l|l|l|l|l|l|l|}
\hline \multicolumn{2}{|c|}{} & \multicolumn{2}{|l|}{ Outcome } & Sensitivity & Specificity & PPV & NPV & Accuracy \\
\cline { 2 - 9 } & Abnormal & Normal & & & & & \\
\hline $\begin{array}{l}\text { Central brain } \\
\text { injury }\end{array}$ & Yes & $\mathbf{2 1}$ & $\mathbf{1 1}$ & 87.5 & 57.69 & 65.63 & 83.33 & 72.6 \\
\cline { 2 - 9 } & $\mathbf{N o}$ & $\mathbf{3}$ & $\mathbf{1 5}$ & & & & \\
\hline Chi square(p) & $\mathbf{1 1 . 0 6 3 ( 0 . 0 0 0 8 )}$ & & & & & \\
\hline
\end{tabular}

Table (3): Agreement between outcome and central brain injury

The sensitivity of peripheral brain injury to predict a poor outcome is $45.83 \%$; the specificity is $70.37 \%$, the positive and negative predictive values are $57.89 \%$ and $59.38 \%$; respectively. Finally, the overall accuracy is $58.1 \%$.

\section{Discussion}

In our study, the mean age of the study group was 19.8 days, with a range from 6 days to one month (30 days), around two-thirds of the cases (64\%) were males, and $36 \%$ were females, the mean gestational age of the study group was 17.92 weeks, with around $40 \%$ of the cases had 38 weeks' gestational age. The mean weight of the study neonates was $3.7 \mathrm{Kg}$, with a range of $3-4 \mathrm{Kg}$. The vast majority of our cases $(78 \%)$ were from rural areas, while only $22 \%$ were from urban regions. The majority of their cases (20 out of 29 cases) were males, and the mean birth weight was $3.24 \mathrm{Kg}$ (with a range from 2.05 to 4.48 $\mathrm{Kg}$ ). On the other hand, in the study of Lally et al.; the mean age was 23 months, mean birth weight was $3.4 \mathrm{~kg}$, mean gestational age in their study was 39.9 weeks, with 216 infants undergoing $72 \mathrm{~h}$ of moderate hypothermia $\left(33.5^{\circ} \mathrm{C}\right)$, six infants being rewarmed before $72 \mathrm{~h}$, and one infant being rewarmed after $96 \mathrm{~h}$ due to local clinical decisions (8). 
In the current study, $46 \%$ of the cases had Sarnat stage I, 40\% had Sarnat stage II while only $14 \%$ had stage III, around half of the cases (48\%) had mild Thompson score, $38 \%$ had a moderate score and $14 \%$ had a severe score. According to the Egyptian study of Noaman et al.; $50 \%$ of the cases have Sarnat stage I, 36.7\% had stage II and 13.3 had stage III (9).

We found that 7 neonates of our study had normal MRI findings with normal neurological outcome. Others showed different patterns of injury; 24 neonates showed central pattern ( $\mathrm{T} 1 \mathrm{hyperint}-$ ensity of the putamina and thalami with loss ofT1 hyperintensity of the posterior limbs of the internal capsules and some cases had shown decreased signal intensity of both thalami in T2WI), 11 cases of them had normal outcome at the time of early discharge and 13 neonates had an abnormal neurological outcome. We found that 11 neonates had shown a peripheral type of injury (cortical, subcortical white matter and or periventricular white matter hyperintense signal in T2W1, FLAIR and or DWI; 5 cases show restriction of diffusion in DWI and presented in early time (Within first 10 days) with or without smudging of the grey-white matter interface at the watershed regions). We found that 3 neonates of 11 had normal neurological outcomes at the time of discharge and the other 8 neonates had abnormal neurological outcomes at the time of discharge. While the other 8 neonates had shown both central and peripheral patterns of brain injury and all of them had an abnormal neurological outcome. In our study, $64 \%$ of our cases had central brain injury; either alone $(48 \%)$ or combined with peripheral injury (16\%); while peripheral injury alone was seen in $22 \%$ of the cases. Seven cases (14\%) had no brain injury. According to Lally et al.; 31 infants $(16 \%)$ had moderate or severe disability, including one death (10). In the study of Gue et al.; out of the total of 29 cases, 13 cases had no MRI abnormalities; 10 had minor to moderate abnormalities; 4 cases had severe abnormalities; with two mortality cases (one after 6 days and one after 12 days) (11). According to Noaman et al.; the MRS showed that $56.7 \%$ of the cases had mild hypoxic-ischemic encephalopathy (HIE); 30\% had moderate HIE and 13.3 had severe HIE, a picture which was non significantly different from Sarnat classification of the same cases (12).

We found that nearly all cases $(90 \%)$ had abnormal findings on MRI spectroscopy. We found that $48 \%$ of the cases had abnormal neurological status at the time of discharge. $100 \%$ of cases with abnormal outcome showed abnormal MRI spectroscopic findings, compared to $80.8 \%$ among those with the normal outcome. The difference was statistically significant with a p-value of 0.031

Although we found that there was a highly significant relationship between the type of brain injury and outcome, with a p-value of less than 0.001 . Among those with the abnormal outcome, $54.2 \%$ had a central brain injury, $33.3 \%$ had both central and peripheral injuries, 12.5 had only peripheral injuries; while none of them had a normal brain. On the other hand, among those with the normal outcome, $42.3 \%$ had a central brain injury, $30.8 \%$ had a peripheral injury, $26.9 \%$ had the normal brain, and nonhad combined central and peripheral injuries.

In this study, the sensitivity of MRI to predict a poor outcome is $100 \%$. However, the specificity is very low $(19.23 \%)$, the positive and negative predictive values are $53.33 \%$ and $100 \%$; respectively. Finally, the overall accuracy is $59.62 \%$. The sensitivity of central brain injury to predict a poor outcome is $87.5 \%$; the specificity is $57.69 \%$, the positive and negative predictive values are $65.63 \%$ and $83.33 \%$; 
respectively. Finally, the overall accuracy is $72.6 \%$. The sensitivity of peripheral brain injury to predict a poor outcome is $45.83 \%$; the specificity is $70.37 \%$, the positive and negative predictive values are $57.89 \%$ and $59.38 \%$; respectively. Finally, the overall accuracy is $58.1 \%$.

We found 19 cases with mild HIE (low lactate peak); Normal NAA, normal choline, and creatine. We found 19 cases with moderate HIE (intermediate lactate peak), mildly decreased NAA. We found 7 cases with severe (high lactate levels). Decreased NAA significantly and decreased NAA/choline ratio. In injured newborns with HIE, a large quantity of lactate is typically present demonstrates a case of an HIE patient born at term with elevated lactate levels across the brain. We found $48 \%$ of the cases had abnormal neurological status at the time of discharge. $100 \%$ of cases with abnormal outcome showed abnormal MRI spectroscopic findings, compared to $80.8 \%$ among those with the normal outcome. The difference was statistically significant with a p-value of 0.031 .

Only 45 of examined neonates show abnormal lactate peak; Low peak, moderate peak or high peak, reduced NAA, and elevated choline, although normal neonatal MRS usually reveals mildly elevated choline and reduced NAA due to incomplete myelination process. The study of $\boldsymbol{Z h u}$ et al. showed that; differences in $\mathrm{N}$ acetyl-aspartate (NAA)/Cr, NAA/Cho, Lac/Cr, and GLX-a/Cr values between the HIE and control groups were significant. Moreover, the values of Glx-a/Cr and $\mathrm{Lac} / \mathrm{Cr}$ in the moderate and severe HIE groups were higher than those in the mild HIE group (13). Lally et al. reported in their study that sensitivity of MRI was $88 \%$ (95\% CI 70-98) and specificity of $90 \%$ (84-95), in their study, basal ganglia or thalamic. injury on conventional MRI had a sensitivity of $71 \%$ (95\% CI 52-
86) and specificity of $88 \%$ (82-93), their results confirming the results of previous meta-analysis (14). The metaanalysis of Thayyil et al. reported that prognostic utilities of various quantitative cerebral MR biomarkers in neonatal encephalopathy. Thirty-two studies were included that were performed between January 1990 and July 2008. Regarding predicting adverse outcomes, T1WIs during the first 30 days had a pooled sensitivity of $91 \%$ (95\% confidence interval [CI], 8794\%). The pattern of injury identified with T1WI may provide diagnostic and prognostic information for neonatal encephalopathy $\underline{(15,16)}$.

Also, Gue et al. reported that the T1 images in the mild to moderate $(n=13)$ and severe group $(n=11)$ were similar, with punctate hyperintensity in the cortex or subcortical white matter $(\mathrm{P}>0.05)$. Profound lesions in the basal ganglia and thalamus are often associated with serious HIE (17).

Similar to our findings, Cheong et al. reported in their study that metabolite concentrations, in particular [NAA], are the most accurate of the MR spectroscopy measurable in neonatal encephalopathy for assigning prognosis (18). A preliminary study of $\boldsymbol{C a d y}$ on metabolite concentrations in neonates with neonatal encephalopathy documented increases in [Lac] and reductions in [NAA], [Cho], and [Cr] (18), similar to those reported in our study.

\section{Conclusion:}

MR spectroscopy is an accurate, sensitive, and non-invasive method for early detection of perinatal ischemic brain injuries. However, the specificity of this maneuver is very low, especially if used in the prediction of a poor outcome

\section{References:}

1. Martinello K, Hart A, Yap S, Mitra S, Robertson N. Management and investigation of neonatal encephalopathy: 2017 update. Archives of disease in 
childhood Fetal and neonatal edition. 2017;102(4):346-58.

2. Al-Macki N, Miller SP, Hall N, Shevell $\mathrm{M}$. The spectrum of abnormal neurologic outcomes subsequent to term intrapartum asphyxia. Pediatric neurology. 2009;41(6):399-405.

3. van Kooij BJ, van Handel M, Nievelstein RA, Groenendaal F, Jongmans MJ, de Vries LS. Serial MRI and neurodevelopmental outcome in 9- to 10-year-old children with neonatal encephalopathy. The Journal of pediatrics. 2010;157(2):221-7.e2.

4. Forbes KP, Pipe JG, Bird R. Neonatal hypoxic-ischemic encephalopathy: detection with diffusion-weighted MR imaging. AJNR American journal of neuroradiology. 2000;21(8):1490-6.

5. Grant PE, Yu D. Acute injury to the immature brain with hypoxia with or without hypoperfusion. Radiologic clinics of North America. 2006;44(1):6377 , viii.

6. Wijdicks EF, Campeau NG, Miller GM. MR imaging in comatose survivors of cardiac resuscitation. AJNR American journal of neuroradiology. 2001;22(8):1561-5.

7. Clements CM, McNally RS, Conti BJ, Mak TW, Ting JP. DJ-1, cancer- and Parkinson's disease-associated protein, stabilizes the antioxidant transcripttional master regulator Nrf2. Proceedings of the National Academy of Sciences of the United States of America. 2006;103(41):15091-6.

8. Hayes BC, Ryan S, McGarvey C, Mulvany S, Doherty E, Grehan A, et al. Brain magnetic resonance imaging and outcome after hypoxic-ischaemic encephalopathy. The journal of maternalfetal \& neonatal medicine: the official journal of the European Association of Perinatal Medicine, the Federation of Asia and Oceania Perinatal Societies, the International Society of Perinatal Obstet. 2016;29(5):777-82.

9. Cheong JL, Thompson DK, Wang HX, Hunt RW, Anderson PJ, Inder TE, et al.
Abnormal white matter signal on MR imaging is related to abnormal tissue microstructure. AJNR American journal of neuroradiology. 2009;30(3):6238.

10. Xu D, Vigneron D. Magnetic resonance spectroscopy imaging of the newborn brain--a technical review. Semin Perinatol. 2010;34(1):20-7.

11. Guo L, Wang D, Bo G, Zhang H, Tao W, Shi Y. Early identification of hypoxic-ischemic encephalopathy by a combination of magnetic resonance (MR) imaging and proton MR spectroscopy. Experimental and therapeutic medicine. 2016;12(5):2835-42.

12. Norman A, Elshafey R, El-Shahawy A, Rowisha M, El-Batch M, Fakhr-Eldin A. MR spectroscopy, S100B protein, and NSE analysis as early predictors of hypoxic-ischemic encephalopathy. The Egyptian Journal of Radiology and Nuclear Medicine. 2013; 44:309-20.

13. Zhu W, Zhong W, Qi J, Yin P, Wang $\mathrm{C}$, Chang L. Proton magnetic resonance spectroscopy in neonates with hypoxic-ischemic injury and its prongostic value. Translational research: the journal of laboratory and clinical medicine. 2008;152(5):225-32.

14. Lally PJ, Montaldo P, Oliveira V, Soe A, Swamy R, Bassett P, et al. Magnetic resonance spectroscopy assessment of brain injury after moderate hypothermia in neonatal encephalopathy: a prospective multicentre cohort study. The Lancet Neurology. 2019;18(1):3545

15. Thayyil S, Chandrasekaran M, Taylor A, Bainbridge A, Cady EB, Chong WK, et al. Cerebral magnetic resonance biomarkers in neonatal encephalopathy: a meta-analysis. Pediatrics. 2010;125(2):e382-95.

16. de Vries LS, Toet MC. Amplitude integrated electroencephalography in the full-term newborn. Clinics in perinatology. 2006;33(3):619-32, vi. 
17. Cheong JL, Cady EB, Penrice J, Wyatt JS, Cox IJ, Robertson NJ. Proton MR spectroscopy in neonates with perinatal cerebral hypoxic-ischemic injury: metabolite peak-area ratios, relaxation times, and absolute concentrations. AJNR American journal of neuroradiology. 2006;27(7):1546-54.

18. Cady EB. Metabolite concentrations and relaxation in perinatal cerebral hypoxic-ischemic injury. Neurochemical research. 1996;21(9):1043-52. 